\title{
A possible theory for the interaction between convective activities and vortical flows
}

\author{
N. Zhao ${ }^{1}$, X. Y. Shen ${ }^{2}$, Y. H. Ding ${ }^{3}$, and M. Takahashi ${ }^{4}$ \\ ${ }^{1}$ State Key Laboratory of Severe Weather, Chinese Academy of Meteorological Sciences, Beijing, 100081, China \\ ${ }^{2}$ Key Laboratory of Meteorological Disaster of Ministry of Education, Nanjing University of Information Science and \\ Technology, Nanjing 210044, China \\ ${ }^{3}$ National Climate Center, Beijing, 100081, China \\ ${ }^{4}$ Atmosphere and Ocean Research Institute (AORI), University of Tokyo, 5-1-5 Kashiwanoha, Kashiwa, 277-8568, Japan
}

Received: 6 May 2011 - Revised: 26 October 2011 - Accepted: 26 October 2011 - Published: 31 October 2011

\begin{abstract}
Theoretical studies usually attribute convections to the developments of instabilities such as the static or symmetric instabilities of the basic flows. However, the following three facts make the validities of these basic theories unconvincing. First, it seems that in most cases the basic flow with balance property cannot exist as the exact solution, so one cannot formulate appropriate problems of stability. Second, neither linear nor nonlinear theories of dynamical instability are able to describe a two-way interaction between convection and its background, because the basic state which must be an exact solution of the nonlinear equations of motion is prescribed in these issues. And third, the dynamical instability needs some extra initial disturbance to trigger it, which is usually another point of uncertainty. The present study suggests that convective activities can be recognized in the perspective of the interaction of convection with vortical flow. It is demonstrated that convective activities can be regarded as the superposition of free modes of convection and the response to the forcing induced by the imbalance of the unstably stratified vortical flow. An imbalanced vortical flow provides not only an initial condition from which unstable free modes of convection can develop but also a forcing on the convection. So, convection is more appropriately to be regarded as a spontaneous phenomenon rather than a disturbance-triggered phenomenon which is indicated by any theory of dynamical instability. Meanwhile, convection, particularly the forced part, has also a reaction on the basic flow by preventing the imbalance of the vortical flow from further increase and maintaining an approximately balanced flow.
\end{abstract}

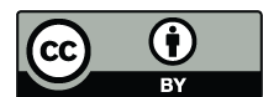

Correspondence to: N. Zhao

(zhaon@cams.cma.gov.cn)

\section{Introduction}

Convective activities have long been a subject of great importance for the study of severe weather. Although applied research toward the understanding of the generation and the roles of convection has considered many relevant aspects concerning latent heating, friction and other physical processes such as the statistical equilibrium theory of Arakawa and Schubert (1974), boundary forcing and surface fluxes, wind-induced surface heat exchange (WISHE), Turbulent Kinetic Energy (TKE) and buoyancy production, etc., purely theoretical studies so far have mainly attributed convection to the dynamical instabilities of the large scale basic state, including static instability and symmetric instability (see, e.g. Holton, 1992; Hoskins, 1974; Xu and Clark, 1985). The mechanisms of these dynamical instabilities give a reasonable explanation to many aspects of convective activities. Meanwhile, many other aspects of convective activities still remain beyond the scope of the instability perspective mainly due to the following three reasons. First, previous instability theories only deal with very simple basic states such as the static state or parallel geostrophic flows with vertical/horizontal shears (Holton, 1992; Pedlosky, 1979; Drazin, 1981), which are too simple cases to have more applications. It is almost impossible to establish the instability theory of a general basic state, not only because of the difficulties in ensuring the existence and finding out the exact solution for a general basic state, but also because of the even more difficult mathematics for the instability of such basic states (see Zhao, 2003 and references therein). Second, convective activities and their spatiotemporal structures are essentially results of the two-way interaction between meso- and synoptic scales (Emanuel et al., 1994; Roode et al., 2004). So, instability with the prescribed basic state as an exact solution of the nonlinear equations of motion cannot be the most suitable

Published by Copernicus Publications on behalf of the European Geosciences Union and the American Geophysical Union. 
description of this relationship between convection and its environment. Last, once the basic state becomes unstable, it does not mean that convection can arise, since an extra initial disturbance is usually needed to trigger the convection. Sometimes, identifying the source of this initial disturbance is itself a difficult problem, because any transient disturbance to the atmosphere has been finally damped after long time evolution and even the existence of such disturbance is hard to determine.

The development of theories on balanced flow and slow manifold in the past decades (for a review on this issue, see e.g. McIntyre, 2000) provides a new possibility for the understanding of convective activities, particularly for overcoming the above three drawbacks of dynamical instability theories. The replacement of the basic states of the instabilities by an approximate and adjustable balanced flow will logically infer the existence of a new mechanism for the spontaneous production of the convection from the balanced flow. This can be viewed as a generalization of the concept of the spontaneous emission of inertia gravity waves by balanced or vortical flow (Lighthill, 1952; Ford et al., 2000). The motivation of the present study is to incorporate convective activities in the framework of the theory of the balanced flow or slow manifold so as to investigate the arising, development and spatiotemporal structure of convection and the conditions of the balanced flow corresponding to these aspects. The paper is arranged as follows. In Sect. 2, by discussing some general properties of convection and the balance/imbalance of vortical flow, we give generalized definitions to the basic concepts associated with convection and its environment. In Sect. 3 we develop a theory for the response of convective activities to the forcing induced by the departure of a vortical flow from balance. Many related issues such as the two-way interaction between convection and vortical flow are discussed there as well. The last section is devoted to a summary and further discussion of related issues.

\section{Generalizations of basic concepts associated with convection}

Before further discussions, some important concepts and associated terminology need to be clarified. We call the environment of convection the basic state or basic flow. The basic state may be roughly defined as the remaining part of motion after convection is removed in some given way. In this definition, the basic flow can be either a strict solution or just an approximate one, with or without the property of balance. In contrast, we need also an appropriate definition of convection to include more complex cases. The following parts of this section are devoted to generalizing these concepts and giving more precise definitions.

\subsection{Basic state, vortical flow, balance and imbalance}

As a generalization of the simple basic state in the previous instability theories such as static and parallel geostrophic flows, we would like to introduce the concept of the balanced flow via that of the slow manifold. The starting point is given by the vorticity equation, divergence equation and thermodynamic equation in $p$-coordinates as below

$$
\begin{aligned}
\frac{\partial \zeta}{\partial t}= & -f \delta-\boldsymbol{V} \cdot \nabla \varsigma-\omega \frac{\partial \varsigma}{\partial p}-\varsigma \delta+\boldsymbol{k} \cdot\left(\frac{\partial \boldsymbol{V}}{\partial p} \times \nabla \omega\right) \\
\frac{\partial \delta}{\partial t}= & f \varsigma-\nabla^{2} \phi-\boldsymbol{V} \cdot \nabla \delta-\omega \frac{\partial \delta}{\partial p} \\
& -\frac{1}{2}\left(\delta^{2}+a^{2}+b^{2}-\varsigma^{2}\right)-\frac{\partial \boldsymbol{V}}{\partial p} \cdot \nabla \omega
\end{aligned}
$$

$\frac{\partial}{\partial t}\left(\frac{\partial \phi}{\partial p}\right)=-\sigma \omega-\boldsymbol{V} \cdot \nabla\left(\frac{\partial \phi}{\partial p}\right)$,

where $\delta$ is the horizontal divergence, $\varsigma$ the vertical component of vorticity, $\boldsymbol{V}$ the horizontal wind with zonal component $u$ and meridional component $v, \omega$ the vertical wind and $\phi$ the potential height. The Coriolis-parameter $f$ has a typical value of $10^{-4} \mathrm{~s}^{-1}$ for mid-latitudes. The stability parameter $\sigma \equiv-\mathrm{RT}_{0} p^{-1} d \ln \theta_{0} / d p$ for the isobaric system is approximately a constant, and $\theta_{0}$ is the potential temperature corresponding to the basic state temperature $T_{0}$. In addition, $a=\partial u / \partial x-\partial v / \partial y, b=\partial v / \partial x+\partial u / \partial y$ are deformations of the horizontal wind field. From the continuity equation, vertical velocity is related to the divergence $\delta$ by $\omega=\int_{0}^{p} \delta d p$. For simplicity, in the equations above the hydrostatic assumption is made, and the advection of the Coriolis-parameter $f$, which gives rise to the $\beta$-effect and the related generation of Rossby waves, is neglected as well. These simplifications specify the range of spatial scales in the present study. Since a small-scale convection cell has strong deviations from the hydrostatic balance, the equations above are more applicable to a meso-scale convection system than to an individual cell within it. On the other hand, the spatial scale should not be too large, so that the $\beta$-effect is negligible. Accordingly, the applicable spatial scales of the equations range from 100 to $1000 \mathrm{~km}$. So, by "convective activities" we primarily mean meso-scale systems in this paper.

A balanced flow can be defined by letting $\delta=0$ in Eq. (1b). The so-called balance equation then is

$f \varsigma-\nabla^{2} \phi-\frac{1}{2}\left(a_{\varsigma}^{2}+b_{\varsigma}^{2}-\varsigma^{2}\right)=0$.

Hereafter, the subscript $\varsigma$ and $\delta$ denote the pure contributions from the vortical and divergent component of the flow, respectively. Equation (2) indicates some certain way of balance between horizontal wind and geopotential height field. 
On the other hand, $\delta=\delta(\varsigma, \phi) \equiv 0$ also defines a hypersurface called the slow manifold in the phase space spanned by $(\varsigma, \delta, \phi)$. It can be viewed as a special case of the slow manifold defined by Leith (1980) and Lorenz (1980). On this slow manifold, the evolution of the system (1) is reduced to

$\frac{\partial \varsigma}{\partial t}=-\boldsymbol{V}_{\varsigma} \times \nabla \varsigma$

$\frac{\partial}{\partial t}\left(\frac{\partial \phi}{\partial p}\right)=-\boldsymbol{V}_{\varsigma} \times \nabla\left(\frac{\partial \phi}{\partial p}\right)$,

i.e. an advection process of the relative vorticity (when $f$ is constant) and the hydrostatic approximated temperature induced by the vortical component of velocity. The balanced flow defined in this way is a purely vortical flow. Obviously, basic states of static flow, parallel geostrophic flow and axisymmetric gradient flow are just particular cases of this balanced flow. Just like deviations of the geostrophic equilibrium, deviations of this general balanced flow can be on the one hand fast gravity waves, or on the other hand a slow and forced secondary divergent motion $\delta$ restoring the balance.

Slow manifold or balanced flow has been a central concept for the understanding of many aspects of the atmospheric dynamics. Much research has been devoted to this subject since it was proposed by Leith (1980) and Lorenz (1980), among which was the general discussion about the existence of a slow manifold for realistic atmospheric flows starting with Lorenz (1986). The most important result of this research was the discovery that the slow manifold is not an exact invariant manifold. It can only exist as a modified concept of so called slow quasi-manifold or fuzzy manifold (Lorenz, 1986, 1987, 1992; Jacobs, 1991; Vautard and Legras, 1986; Vallis, 1996; Vanneste and Yavneh, 2004; Warn and Menard, 1986; Warn et al., 1995; Warn, 1997; Ford, 2000), which means that balanced flow, to some extent, is just an approximation except for some particular cases such as parallel geostrophic flows and axisymmetric gradient flows. There is also now strong experimental evidence that the slow manifold is not an invariant manifold (Williams et al., 2008).

Since a much stronger condition $\delta=0$ is imposed on the primitive equations in Eq. (1), the balance system of Eqs. (2) and (3) in this paper are neither exactly analogous to the balance equations by Charney (1955) which permit the spurious nonphysical solutions noted by Moura (1976) nor to the slow equations by Lynch (1989) in which the spurious solutions are absent. As the slow manifold defined in the present way usually can not exist as an exact invariant manifold, the balance system of Eqs. (2) and (3) may also permit spurious nonphysical solutions. But the nonphysical parts of the solutions may be small enough to be neglected and the nonphysical solutions are roughly physical ones, if the slow manifold remains to be a quasi-manifold.

It is also well recognized that the departure from the exact balance is associated with the activities of inertia gravity waves or the spontaneous emission (Ford et al., 2000).
However, convection, which may be another important phenomenon associated with this loss of balance, has not been investigated theoretically so far. In our following studies on meso-scale disturbance such as convective activities, the basic state can be selected as the vortical flow, no matter whether or not it is an exact balanced flow. In this case, the vortical component $\varsigma$ together with $\phi$ is viewed as the basic state, while the divergent component $\delta$ is the disturbances about it. This idea is more clearly seen by a mathematical definition as below. Let $\boldsymbol{S}$ denote the phase state of the dynamical system (1), $\boldsymbol{S}_{0}$ the basic state and $\boldsymbol{S}^{\prime}$ the disturbance, so

$\boldsymbol{S} \equiv\left(\begin{array}{l}\delta \\ \varsigma \\ \phi\end{array}\right)=\left(\begin{array}{l}\delta \\ 0 \\ 0\end{array}\right)+\left(\begin{array}{l}0 \\ \varsigma \\ \phi\end{array}\right) \equiv \boldsymbol{S}^{\prime}+\boldsymbol{S}_{0}$

regardless whether or not the basic state $\boldsymbol{S}_{0}$ satisfies the balance Eq. (2). The conventional theory of balanced flow divides the atmospheric motions into two classes: highfrequency inertia-gravity waves (phase speeds up to hundreds $\mathrm{ms}^{-1}$ and large divergence) and large-scale lowfrequency flow (phase speeds of the order of ten $\mathrm{ms}^{-1}$, periods of few days, vortical flow). However, the convective scales are in between. So dividing atmospheric motions into divergent and vortical flow rather than into high- and lowfrequency seems more essential in the understanding of the relationship between convection and balanced flow.

\subsection{Effects of balanced/imbalanced vortical flows on convection}

Equation (1) can be reduced to one equation for $\delta$

$$
\frac{\partial^{2} \delta_{p p}}{\partial t^{2}}+\sigma \nabla^{2} \delta+f^{2} \delta_{p p}+\Im(\delta)-\ell_{\varsigma, \phi} \delta=\Re(\varsigma, \phi) .
$$

Here, the subscript $p$ denotes the partial derivative with respect to $p . \Im(\delta)$ is the nonlinear term of the disturbance $\delta$. As no analytical solution of Eq. (4) can be gained in the nonlinear regime, it will be omitted in the following discussions where $\delta$ can be assumed to be small enough, not only because we just care about the triggering stage of the convection, but also because $\delta$ is usually far smaller than $\zeta$ as will be pointed out later in the next section. Finally,

$$
\begin{aligned}
\ell_{\varsigma, \phi} \delta= & {\left[-f \nabla \varsigma \cdot \boldsymbol{V}_{\delta}-f \frac{\partial \varsigma}{\partial p} \omega-f \varsigma \delta+f \boldsymbol{k} \cdot\left(\frac{\partial \boldsymbol{V}_{\varsigma}}{\partial p} \times \nabla \omega\right)\right]_{p p} } \\
& -\left[\boldsymbol{V}_{\varsigma} \cdot \nabla \delta+\left(a_{\varsigma} a_{\delta}+b_{\varsigma} b_{\delta}\right)+\frac{\partial \boldsymbol{V}_{\varsigma}}{\partial p} \cdot \nabla \omega\right]_{t p p} \\
& +\nabla^{2}\left[\nabla\left(\frac{\partial \phi}{\partial p}\right) \cdot \boldsymbol{V}_{\delta}\right]_{p}
\end{aligned}
$$

is the linear operator acting on $\delta$, while 


$$
\begin{aligned}
\mathfrak{R}(\varsigma, \phi)= & -\frac{1}{2}\left[\left(a_{\varsigma}^{2}+b_{\varsigma}^{2}-\varsigma^{2}\right)_{t}\right]_{p p}-f\left(\boldsymbol{V}_{\varsigma} \times \Delta \varsigma\right)_{p p} \\
& +\Delta^{2}\left[\boldsymbol{V}_{\varsigma} \times \Delta\left(\frac{\partial \rho}{\partial p}\right)\right]_{p}
\end{aligned}
$$

is the inhomogeneous term which depends only on the basic state $(\varsigma, \phi)$. The subscript $t$ denotes the partial derivative with respect to $t$. The physical meaning of $\Re(\varsigma, \phi)$ is related with the so-called omega equation and will be discussed in detail in the next section.

If the basic state $(\varsigma, \phi)$ is an exactly balanced flow, then it is also the exact solution of Eq. (1). From Eqs. (3) and (6), we have $\Re(\varsigma, \phi)=0$. Then Eq. (4) becomes

$\frac{\partial^{2} \delta_{p p}}{\partial t^{2}}+\sigma \nabla^{2} \delta+f^{2} \delta_{p p}-\ell_{\varsigma, \phi} \delta=0$.

This is a problem of stabilities including static instability and symmetric instability when the basic state is a static flow and parallel geostrophic flow, respectively. It follows that for the exactly balanced flow, convection can only be attributed to the instabilities of the balanced flow. For example, when the balanced flow is the parallel geostrophic flow, and only symmetric disturbance is considered, Eq. (7) can be rewritten as

$\frac{\partial^{2} \delta_{p p}}{\partial t^{2}}+N^{2} \delta_{y y}-2 S^{2} \delta_{y p}+F^{2} \delta_{p p}=0$.

Here, $N^{2}=\sigma, \quad S^{2}=f U_{p}, F^{2}=f\left(f+U_{y}\right)$, and $U$ is the $x$-oriented basic flow. It can be proven that the criterion for the symmetric instability is

$q=F^{2} N^{2}-S^{4}<0$ when $N^{2}>0$.

Nevertheless, the exactly balanced flows are just a few of very particular cases as mentioned above. Under ordinary circumstances, the basic state $(\zeta, \phi)$ may more or less remain apart from this exact balance. So, usually we have the inhomogeneous term $\Re(\varsigma, \phi) \neq 0$, which appears as some external forcing on the convection from the basic state $(\varsigma, \phi)$. Consequently, besides producing instabilities, the impact of vortical flow on convection can also be attributed to a forcing by the imbalance of vortical flow. However, $\Re(\varsigma, \phi)$ does not directly depend on this departure. Rather, as shown in Eq. (6), it depends on the spatiotemporal derivatives of each of the three individual terms (or their advections) in Eq. (2) which cancel each other only in the case of exact balance. As a result, the forcing is not determined by the imbalance of the three terms of Eq. (2) but by the imbalance of spatiotemporal derivatives of them (or their advections) in Eq. (6). This fact means that far departure from the balance does not need to indicate a stronger forcing than a small departure and that the forcing by the vortical flow can be very complex. Even so, $\Re(\varsigma, \phi)$ can still be used to measure the departure of the vortical flow from the balance, because at least the distinction between the balanced and imbalance flows is reflected well by $\Re(\varsigma, \phi)=0$ or $\Re(\varsigma, \phi) \neq 0$, respectively. A physical explanation of this imbalance forcing will be given in Sect. 3 .

\subsection{Reconsideration of the definition of convection}

As mentioned above, if the basic states are purely balanced flows, convection can be defined traditionally as the vertical motion arising from the instabilities of these balanced flows. However, the loss of balance of the vortical flow always yields an inhomogeneous term $\Re(\varsigma, \phi) \neq 0$ to Eq. (7), that is

$$
\frac{\partial^{2} \delta_{p p}}{\partial t^{2}}+\sigma \nabla^{2} \delta+f^{2} \delta_{p p}-\ell_{\zeta, \phi} \delta=\Re(\varsigma, \phi) .
$$

In this case, since the basic state is no longer the exact solution, the definition of its stability becomes problematic and so does the definition of convection. Consequently, we need to reconsider the definition of convection and give a more general one to include imbalance cases.

The general solution of the linear Eq. (10) should be the superposition of both the homogeneous solution $\delta_{1}$ satisfying only homogeneous part of Eq. (10), and the inhomogeneous solution $\delta_{2}$ satisfying the whole equation of (10),

$\delta=\delta_{1}+\delta_{2}$.

It is easy to see that the homogeneous part and its solution $\delta_{1}$ behave like a problem of stability, no matter whether or not $(\varsigma, \phi)$ is an exactly balanced flow with $\Re(\varsigma, \phi)=0$. So we can propose an apparent stability problem like Eq. (7) for $\delta_{1}$ even when $\Re(\varsigma, \phi) \neq 0$. As a result, convection is definitely associated with this kind of apparent instability (Kelvin-Helmholtz instability, inertia instability, or symmetric instability). On the other hand, the inhomogeneous solution $\delta_{2}$ for an unstable homogeneous operator of Eq. (10) may also largely differ from that of a stable one that just yields forced inertia gravity waves. So, both the homogeneous and the inhomogeneous solution contribute to the convective activity when the homogeneous operator is unstable. Consequently, the definition of convection can be generalized as the vertical motion resulting from an unstable basic state given by $(\varsigma, \phi)$, regardless whether or not the basic state is a balanced flow. In other words, this generalized definition regards convection as the results of both apparent instability and forcing of an unstable and imbalanced basic state. There are two key points of this generalized definition of convection, i.e. (1) the basic state $(\varsigma, \phi)$ must be apparently unstable, and (2) it needs not to be balanced flow.

\subsection{Disturbance-triggered or spontaneous convection?}

The triggering mechanisms of convection for balanced and imbalanced vortical flows are also different. In the former case, convection is an issue of purely instability which grows 
from some external initial disturbance. We call this kind of convection a disturbance-triggered convection. In the latter case, convection is a result of both apparent instability and response to forcing by an imbalanced basic state. The triggering of the apparent instability does not need an external initial disturbance, because for an imbalanced vortical flow we always have an initial disturbance $\delta_{t=0} \neq 0$. So, imbalance provides not only a forcing but also an initial disturbance from which the apparent instability can develop. Therefore, it is more appropriate to attribute the triggering of convection to the imbalance of the basic state itself rather than to some unknown extra source. We can then call this kind of convection a spontaneous convection. In the linear regime of the development of convection, this imbalance-forced part of convection $\left(\delta_{2}\right)$ cannot interact with free unstable modes of apparent instability $\left(\delta_{1}\right)$. However, as the convection develops into the nonlinear regime, we hypothesize that the nonlinear interaction between them $\left(\delta_{1}\right.$ and $\left.\delta_{2}\right)$ may create an even more complex structure of the convective activity. Even so, the spontaneous nature of the convection remains unchanged.

\section{Convections interacting with vortical flows}

\subsection{Simplification of concepts}

As mentioned above, the linkage between convection and its synoptic background is characterized by both a response to forcing $\Re(\varsigma, \phi)$ and the instability of the basic flow with $\ell_{\zeta, \phi} \delta$ involved. Usually, at synoptic scale, we have a Rossby number $\varepsilon \ll 1$ and

$\left|\frac{\delta}{\varsigma}\right| \leq \varepsilon \ll 1$,

Also, for a meso-scale vortical flow with Rossby number $\varepsilon=$ $O(1)$, we assume that the Froude number $F r$ can be estimated from the barotropic mode by $F r=U / \sqrt{g H}$, where $U \sim 10^{1} \mathrm{~m} \mathrm{~s}^{-1}$ is the scale of wind speed, and $H \sim 10^{4} \mathrm{~m}$ is the vertical scale. In this case, or even for $H \sim 10^{3} \mathrm{~m}$, a much shallower equivalent depth, $F r \ll 1$ can be satisfied very well, so that we have

$\left|\frac{\delta}{\varsigma}\right| \propto \frac{F r^{2}}{\varepsilon} \ll 1$

For details regarding the above scale analysis, we refer to McIntyre (2000) or Ford et al. (2000). Under these conditions, since both $\ell_{\zeta, \phi} \delta$ and $\Re(\varsigma, \phi)$ are quadratic terms, once $\delta$ is small, it can be proven that

$\ell_{\varsigma, \phi} \delta \ll \Re(\varsigma, \phi)$.

Equation (10) is then reduced to

$$
\frac{\partial^{2} \delta_{p p}}{\partial t^{2}}+\sigma \nabla^{2} \delta+f^{2} \delta_{p p}=\Re(\varsigma, \phi)
$$

Without the bilinear term $\ell_{\zeta, \phi} \delta$, the homogeneous part of Eq. (15) is identical to a problem of static instability, which simplifies the concepts and mathematics of the present issues to a great extent. Consequently, the impact of the basic state on convection is merely from the additive forcing $\Re(\varsigma, \phi)$, rather than from the multiplicative forcing $\ell_{\zeta, \phi} \delta$ associated with the apparent instability.

By projecting Eq. (15) on the vertical modes $P_{n}$ defined by the eigen-system

$-\frac{d^{2} P_{n}}{d p^{2}}=\lambda_{n} \sigma P_{n} ; \quad n=0,1,2, \cdots$

satisfying suitable lower and upper boundary conditions, we obtain (see Appendix A)

$\frac{\partial^{2} \delta_{n}}{\partial t^{2}}-c_{n}^{2} \nabla^{2} \delta_{n}+f^{2} \delta_{n}=\Re_{n}(\varsigma, \phi)$.

Here, $c_{n}^{2}=1 / \lambda_{n}$. If $c_{n}^{2}>0$, or the atmosphere is stably stratified, the left hand side of Eq. (17) describes the inertia gravity waves, while the right-hand side is the "source" of these waves. This is the concept of so-called spontaneous emission proposed and well studied in previous works such as Lighthill (1952) and Ford et al. (2000). In the emission Eq. (4), we take the linear term $\ell_{\zeta, \phi} \delta$ to be a source term. However, in the Lighthill/Ford interpretation, it would be on the left-hand side of Eq. (4) and would be regarded as a part of the wave operator. This has implications for the ensuing analysis, because the smallness of the linear term compared to the inhomogeneous term (Eq. 14) is then irrelevant, and what matters is the smallness compared to the other terms in the wave operator. The resulting mathematics is largely different from that of the present analysis. This discrepancy may be understood as follows. Basically, $(\varsigma, \delta, \phi)$ can also be viewed as a small disturbance about the static background. Therefore, by inertia gravity waves, we implicitly mean those under the static background and the wave operator is then just as the left-hand side of Eq. (15). The "linear" term $\ell_{\zeta, \phi} \delta$ is essentially a nonlinear one and should also be a small term even compared to the true linear terms in the wave operator, if weak linearity is assumed.

The spontaneous emission when $c_{n}^{2}>0$ is no longer the topic of our present study. Rather, if the atmosphere is unstably stratified, i.e. $c_{n}^{2}<0$, the left-hand side of Eq. (17) describes the convection, and the right-hand side is viewed as the forcing from the vortical flow, which will be the focus of our following discussion.

The inhomogeneous solution of Eq. (17) or the response to the forcing can be obtained as below. By introducing a new argument $\tau=c_{i} t$ with $c_{i}^{2}=-c_{n}^{2}>0$, Eq. (17) is transformed into a 3-dimensional Helmholtz equation

$\frac{\partial^{2} \delta_{n}}{\partial x^{2}}+\frac{\partial^{2} \delta_{n}}{\partial y^{2}}+\frac{\partial^{2} \delta_{n}}{\partial \tau^{2}}+\frac{f^{2}}{c_{i}^{2}} \delta_{n}=\frac{1}{c_{i}^{2}} \Re_{n}(\varsigma, \phi)$. 
It is highly necessary to point out that the elliptic Eq. (18) is essentially different from the hyperbolic Eq. (18) with $c_{n}^{2}>0$, because the latter is wave equation describing inertia gravity waves while the former describes convection which can not be simply regarded as unstable inertia gravity waves.

\subsection{Analytical solution of convection}

The general solution of Eq. (18) is the sum of its homogeneous and inhomogeneous solutions, corresponding to free modes of convection and forced convection as below, respectively.

a. Free modes of convection

The homogeneous solution of Eq. (18) can be written as $A \exp \left[\left(k_{x} x+k_{y} y+\omega t\right) i\right]$, the growth rate of the unstable mode of which is obtained from the dispersion relation as $\lambda=i \omega=\sqrt{\left(k_{x}^{2}+k_{y}^{2}\right) c_{i}^{2}-f^{2}}$. Obviously, disturbances of small scale tend to grow more rapidly. So, usually these free unstable modes are responsible for the formation of small-scale cells of convection.

\section{b. Forced convection}

The Green's function of Eq. (17) can be obtained from that of Eq. (18) as

$$
G\left(\boldsymbol{r}, \boldsymbol{r}^{\prime}, t, t^{\prime}\right)=\frac{1}{4 \pi} \frac{\exp \left[i \frac{f}{c_{i}} \sqrt{\left|\boldsymbol{r}-\boldsymbol{r}^{\prime}\right|^{2}+c_{i}^{2}\left(t-t^{\prime}\right)^{2}}\right]}{\sqrt{\left|\boldsymbol{r}-\boldsymbol{r}^{\prime}\right|^{2}+c_{i}^{2}\left(t-t^{\prime}\right)^{2}}}
$$

where $\boldsymbol{r}=x i+y j$, and the causality demands $t>t^{\prime}$ (see e.g. Guo, 1979). So, the inhomogeneous solution of Eq. (17) can be written as

$$
\begin{aligned}
& \delta_{n}(\boldsymbol{r}, t)=\frac{1}{4 \pi c_{i}^{2}} \int_{t^{\prime}<t} \int_{-\infty}^{\infty} \int_{-\infty}^{\infty} \Re_{n}\left(\boldsymbol{r}^{\prime}, t^{\prime}\right) \\
& \frac{\exp \left[i \frac{f}{c_{i}} \sqrt{\left|\boldsymbol{r}-\boldsymbol{r}^{\prime}\right|^{2}+c_{i}^{2}\left(t-t^{\prime}\right)^{2}}\right]}{\sqrt{\left|\boldsymbol{r}-\boldsymbol{r}^{\prime}\right|^{2}+c_{i}^{2}\left(t-t^{\prime}\right)^{2}}} d x^{\prime} d y^{\prime} d t^{\prime}
\end{aligned}
$$

The physical meaning is clear: the strength of forced convection in $\boldsymbol{r}$ at arbitrary time $t$ depends on the cumulative influence of the forcing from everywhere and at all times earlier than $t$. The Green's function indicates that the influence of the forcing from the vortical flow is inversely proportional to the spatio-temporal distance, which means that the overall spatio-temporal structure of the forced convection is similar to that of the forcing $\Re(\varsigma, \phi)$. If the structure of the vortical flow is movable, then so is the overall structure of the forced convection associated with it. On the other hand, as indicated by the spatio-temporal structure of the Green's

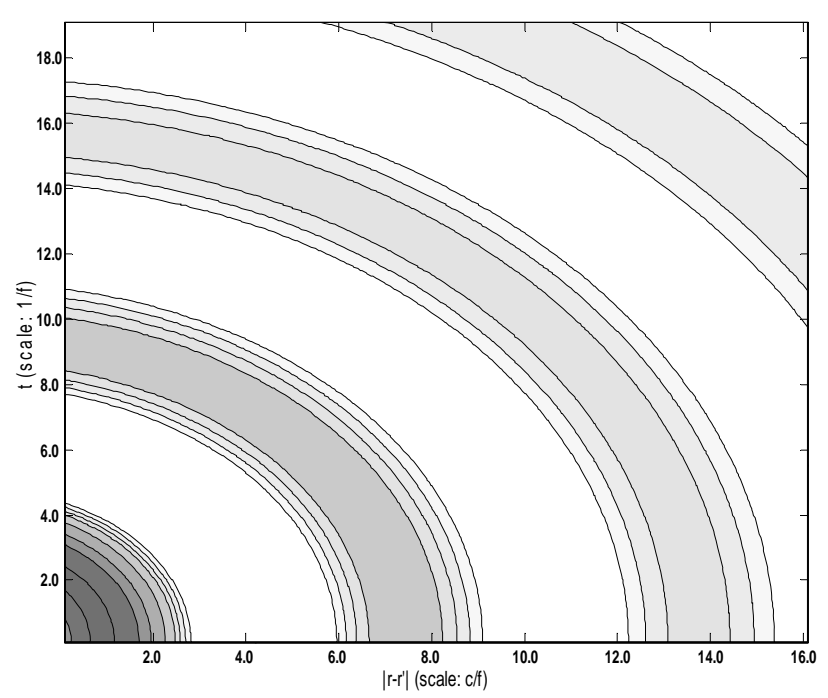

Fig. 1. Spatio-temporal structure of the Green's function (19) (contour lines in divergent regions are not shown). The figure shows that a pulsation at time $t^{\prime}$ of the forcing located at $\boldsymbol{r}^{\prime}$ can induce convective structures around (shadow areas), and that these structures will move towards the "source" at $\boldsymbol{r}^{\prime}$.

function in Fig. 1, the fine structures reflect the numerator of the Green's function (19). It shows that convective structures induced by the pulsation at time $t^{\prime}$ of forcing located at $\boldsymbol{r}^{\prime}$ will move toward the "source" at $\boldsymbol{r}^{\prime}$. We suppose that this is a universal property of forced convection and that its structures tend to approach that the centers with the strongest forcing. This structure of the Green's function solution suggests that a spatial pattern of forced convection can be generated instantly at infinite distance from the source. However, as indicated by the denominator of the Green's function, this structure decays rapidly with the distance from the source. So, in the real world, such pattern can only be expected to be observed in the adjacent region of the source. To illustrate the effect of the cumulation of forcing at different places and times as indicated by Eq. (20), Fig. 2 gives the spatio-temporal structure of the superposition of the responses to two pulsations of forcing at $(x, t)=(0,0)$ and $(x, t)=(10,-2)$. A more complex structure than that in Fig. 1 can be found due to this superposition.

\section{c. Scale analysis of convective activities}

The basic structure of the Green's function indicates that meso-scale forcing gives rise to forced convection of meso-scale, while large-scale forcing gives rise to forced convection of large-scale. However, the numerator part of the Green's function gives a description of the effect of the inertia oscillation on the forced convection. Very complex oscillating/propagating structures may be embedded in (or superposed to) the overall structure 


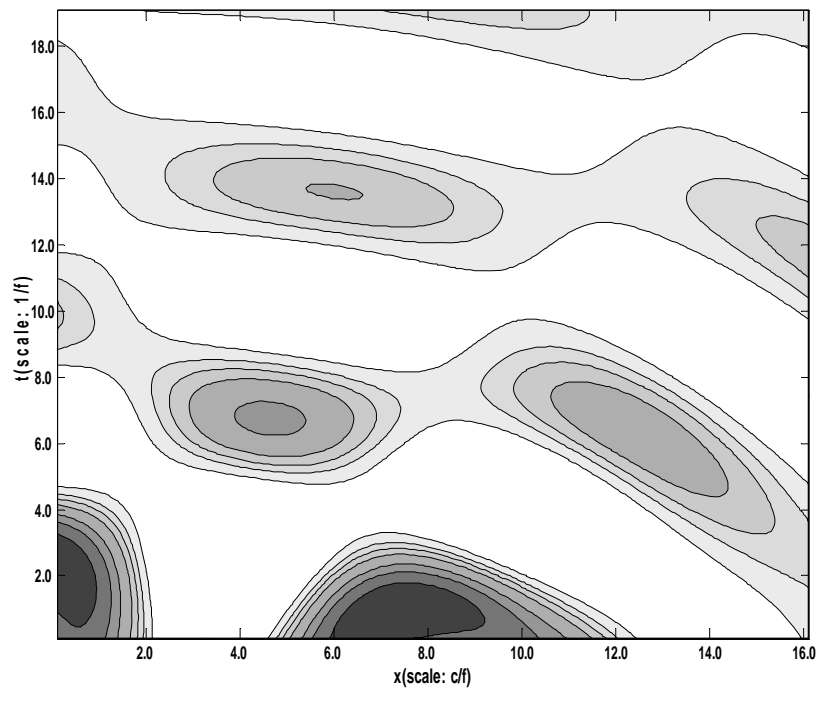

Fig. 2. To illustrate the effect of the superposition of forcings at different places and times as indicated by Eq. (20), the figure shows the spatio-temporal structure of the superposition of the responses to two pulsations of forcing at $(x, t)=(0,0)$ and $(x, t)=(10,-2)$ (contour lines in divergent regions are not shown). A more complex structure can be found due to this superposition.

induced by the forcing of the vortical flow, and are essentially different from the inertia gravity waves.

The spatial scale of such an oscillating/propagating structure is $c_{i} / f$. Since $f=10^{-4} \mathrm{~s}^{-1}, c_{i} / f$ can either be very small or very large, depending on the static instability (or the imaginary phase speed $c_{i}$ ). This structure can be viewed as being embedded in the synoptic scale system when $c_{i}$ is small enough, otherwise it can also be comparable to the synoptic scale when $c_{i}$ is large. However, this is just the case in the situation of a vortical flow of synoptic scale. For a vortical flow of meso-scale, Eq. (13) demands the Froude number $F r \propto U / c_{i} \ll 1$, which gives a limitation to the lower bound of $c_{i}$. In order for the existence of Eq. (18), $c_{i}$ must be large enough, otherwise the multiplicative forcing $\ell_{\zeta, \phi} \delta$ becomes too complex in form and cannot be omitted and leads to a mathematical difficulty beyond the capability of the present study. In this case, the meso-scale vortical flow can induce forced convection with scales larger than the vortical flow itself. In fact, following Ford (2000), we can assume the forcing of the vortical flow to be confined to a small region with diameter $L$. If the scale of the wind speed of the vortical flow is $U$, then the scale of temporal variations is $L / U$. Out of this small region, the growth rate of the free mode of convection can be estimated by the dispersion relationship, i.e. $\lambda=\sqrt{c_{i}^{2} k^{2}-f^{2}}$, where $k$ is the wavenumber. We can assume that it is proportional to
$L / U$, or the time scale of the variation of the vortical flow as the source of forcing. So the scale of forced convection is $2 \pi L / F r \gg L$ when $F r \ll 1$.

Anyway, the scales discussed above just apply to forced convection by the imbalanced vortical flow. Free modes of convection represented by the unstable homogeneous solutions are another important factor that contributes to the spatial scales of the convective activities. Consequently, multiple spatial scales of convective activities are caused by the following three factors: (1) the scale of the imbalance of the vortical flow, (2) the scales of the inertial oscillation and (3) the scales of the unstable free modes of convection. Since unstable free modes of convection tend to select structures with the smallest scales and are embedded in the forced convection, convective activities always appear as the former modulated by the latter.

\subsection{Two-way interaction between convection and vortical flow}

In addition to the response of convection to the forcing induced by the imbalance of vortical flow as mentioned above, Eq. (15) is actually a problem of two-way interactions between convective activities and the basic flow as well. The convective activities can act on the basic flow and contribute to its adjustment. In principle, this interaction between convection and its basic state is described by Eq. (15), although this kind of two-way interaction can never be dealt with in the framework of dynamical instabilities of basic flows.

Generally speaking, the action of the convection on the basic flow seems far more complex to describe. In the present study we would like to address this issue mathematically as below. According to the Fredholm alternative (see any text book on partial differential equation, e.g. Haberman, 2003), the solvability of Eq. (15) requires its inhomogeneous term $\Re(\varsigma, \phi)$ to be orthogonal to the homogeneous solution $\delta_{0}$, or

$<\delta_{0}, \Re(\varsigma, \phi)>=\int_{\Omega} \delta_{0}^{*} \Re(\varsigma, \phi) d \Omega=0$.

Here, $<,>$ is an inner product properly defined over some spatiotemporal domain $\Omega, \delta_{0}$ is the homogeneous solution of Eq. (15) while $\delta_{0}^{*}$ is its adjoint solution. This constraint on $\Re(\varsigma, \phi)$ means that the departure from balance is confined to merely some very special ways. It can then be explained as the action of convection on its basic flow. Since the homogeneous solution $\delta_{0}$ represents all possible free modes of convection, this reaction adjusts the basic flow in a particular way so that the resulting forcing $\Re(\varsigma, \phi)$ has no projection on any free mode of convection. As these free modes of convection are all growing modes and can represent almost all possible ways of growth with time, Eq. (21) implies that $\Re(\varsigma, \phi)$ cannot have any infinitely growing component, 
which may be essentially a limitation to the further intensification of the forcing $\Re(\varsigma, \phi)$, or roughly, a measure for the imbalance. So, the imbalance of the vortical flow cannot increase infinitely and an approximately balanced flow can be maintained.

A more physical explanation for this action of convection on the basic flow can be obtained from an approximate analysis of the Green's function (19). The inhomogeneous solution of convection far apart from the "source" of the imbalance can be estimated by setting the "source" at $\boldsymbol{r}^{\prime}=0$ and $t^{\prime}=0$ in the Green's function. So, we have

$\delta_{n}(\boldsymbol{r}, t) \propto \operatorname{Re}\left(a e^{i \theta}\right)=a \cos (\theta)$,

where the "phase" is given by

$\theta=\frac{f}{c_{i}} \sqrt{|\boldsymbol{r}|^{2}+c_{i}^{2} t^{2}}$

The amplitude $a$ varies slowly with $t$ and $\mathrm{r}$ and is assumed to be constant. On the other hand, out of the "source" region, we have $\Re_{n}(\varsigma, \phi)=0$, so the governing equation should be

$\frac{\partial^{2} \delta_{n}}{\partial t^{2}}+c_{i}^{2} \nabla^{2} \delta_{n}+f^{2} \delta_{n}=0$

although the inhomogeneous solution is considered. Multiplying Eq. (24) by $\partial \delta_{n} / \partial t$, we have the following conservation law of Eq. (24)

$\frac{\partial}{\partial t}\left(\frac{1}{2} \delta_{n t}^{2}-\frac{1}{2} c_{i}^{2}\left|\nabla \delta_{n}\right|^{2}+\frac{1}{2} f^{2} \delta_{n}^{2}\right)+\nabla \cdot\left(c_{i}^{2} \delta_{n t} \nabla \delta_{n}\right)=0$.

By substituting Eqs. (22) and (23) into Eq. (25) and integrating from $\theta=0$ to $\theta=2 \pi$, we rewrite Eq. (25) as

$$
\frac{\partial E}{\partial t}+\nabla \cdot \boldsymbol{F}=0
$$

Here,

$E=\frac{f^{2} c_{i}^{2} t^{2} a^{2}}{2\left(|\boldsymbol{r}|^{2}+c_{i}^{2} t^{2}\right)}$

$\mathbf{F}=\frac{f^{2} c_{i}^{2} t a^{2}}{2\left(|\boldsymbol{r}|^{2}+c_{i}^{2} t^{2}\right)} \boldsymbol{r}$

are the energy density and flux, respectively. The group velocity which indicates the wave energy transportation can be obtained by

$\boldsymbol{c}_{g}=\frac{\mathbf{F}}{E}=\frac{1}{t} \boldsymbol{r}$.

It clearly demonstrates that outward and temporally decaying energy transportation from the "source" accompanies the imbalance, which will essentially reduce the convection or $\delta$ and tends to maintain the balance of the basic flow. The fact that the group velocity goes to infinity when $t$ goes to 0 does not need to mean that there is no point unaffected by the "source", because the energy density $E$ as well as the flux $\boldsymbol{F}$ vanish, i.e. there is no transport of energy. On the contrary, the local phase speed is given by

$\boldsymbol{c}=\frac{-\partial \theta / \partial t}{\partial \theta / \partial x} \boldsymbol{i}+\frac{-\partial \theta / \partial t}{\partial \theta / \partial y} \boldsymbol{j}=-\frac{c_{i}^{2} t}{|\boldsymbol{r}|^{2}} \boldsymbol{r}$,

This phase propagation toward the "source" has been demonstrated in Fig. 1. So, we can conclude that the two-way interaction between convection and its basic state is characterized by the following process: the imbalance of the basic flow generates convection, while the convection suppresses the further increase of this imbalance in turn.

\subsection{Physical explanation and observational evidences}

In order to identify spontaneous convection as described above in the real world, two fundamental aspects, i.e. unstable stratification and imbalance of the basic state should be observed simultaneously. The unstable stratification generates small cells of convection, while the imbalance gives a larger-scale modulation with spatio-temporal structures indicated by Eq. (20). In fact, since no exactly balanced flow can be found in the atmosphere and unstable stratification is also very common, most of the convective activities have something to do with this spontaneous convection.

For the purpose above, we need also an explanation of the physical meaning of the imbalance forcing $\Re(\varsigma, \phi)$. If the vertical or horizontal structures of the terms in brackets in $\Re(\varsigma, \phi)$ are approximately sine or cosine functions, the imbalance forcing can be viewed as the result of $\left(a_{\varsigma}^{2}+b_{\varsigma}^{2}-\varsigma^{2}\right)_{t}$ (nonsteady processes of the vortical flow), $\boldsymbol{V}_{\varsigma} \cdot \nabla_{\zeta}$ (vorticity advection) and $\left[\boldsymbol{V}_{\zeta} \cdot \nabla\left(\frac{\partial \phi}{\partial p}\right)\right]_{p}$ (difference of temperature advections between upper and lower levels). The physical interpretations of these terms are also clear: both local changes of vorticity/deformation with time and vorticity advection can result in changes of the pressure so as to maintain the balance, while the changes of the pressure gradient can cause convergence/divergence in turn. Similarly, temperature advection results in some changes of pressure, while the resulting changes of the pressure gradient can also generate convergence/divergence. These are usually the situations in synoptic systems of various scales such as fronts, jets, troughs, ridges and eddies. These systems are subjected to departures from balanced flows such as geostrophic and gradient flows. For example, a front is associated with a difference of temperature advection between upper and lower level. Moving trough, ridges and eddies of various scales cause vorticity advection, and local changes of deformation are related to the speedup/slowdown of meso-scale jets. All these phenomena may cause imbalances of vortical flows. So, if these systems become unstably stratified and convections arise within them, the convection resulting from these imbalance forcings can be regarded as observational evidences of spontaneous 


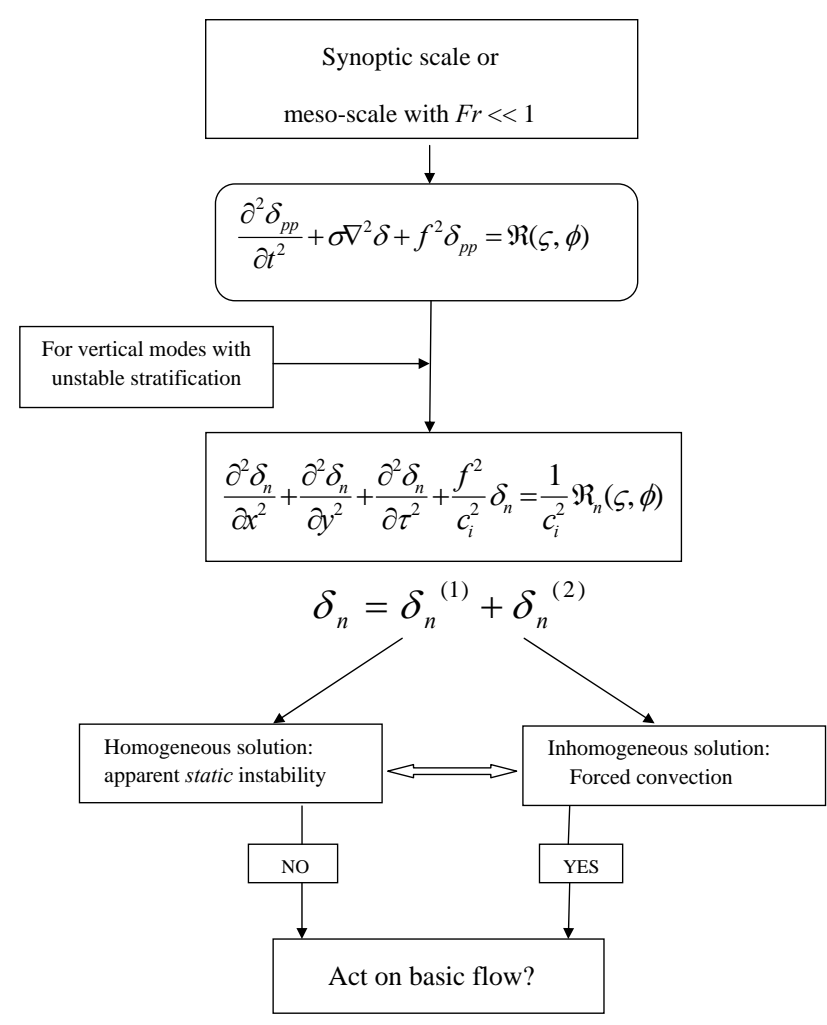

Fig. 3. A schematic overview about the different cases discussed in Sect. 3.

convection. A particular case for convection without large or meso-scale synoptic systems accompanied is daytime heating on a flat and homogeneous surface. In this case, the basic flow is nearly balanced and $\Re(\varsigma, \phi)$ remains very small. Once the stratification of the atmosphere becomes unstable, the unstable free modes dominate over the forced part of convection. Although imbalance may be too weak to generate a noteworthy part of convection, it can still provide an initial disturbance from which instability develops spontaneously. Although the forcing terms are very similar to those of the well-known $\omega$ equation (see, e.g. Holton, 1992), it is worthy to mention that the forced part of convection is essentially different from the issue of vertical motion generated from vortical flow as described by the $\omega$ equation. If the atmosphere is stably stratified $(\sigma>0)$, the $\omega$ equation is an elliptic equation. To the leading order, it describes the spontaneous emission of inertia gravity waves in the "source" region. However, if the atmosphere is unstably stratified $(\sigma<0), \omega$ equation becomes a hyperbolic equation and usually not to be used for the diagnosis of the vertical motion. So, the vertical motion for $\sigma<0$ remains unclear so far, and the present concept of forced convection can't be attributed to the conventional vertical motion. In other words, it is the way of response rather than the form of forcing that is different. A schematic overview about the different cases discussed in this section is given in Fig. 3.

\section{Conclusions and discussions}

The present study suggests that convective activities can be recognized in the perspective of their interaction with the vortical flow. It has been demonstrated that convective activities can be regarded as the superposition of free modes of convection and the response to the forcing induced by the imbalance of the unstably stratified vortical flow. An imbalanced vortical flow provides not only an initial condition from which unstable free modes of convection can develop but also a forcing on the convection. Soconvection is more appropriately to be regarded as a spontaneous phenomenon rather than a disturbance-triggered phenomenon which is indicated by any theory of dynamical instability. Meanwhile, convection, particularly the forced part, has also a reaction on the basic flow by preventing the imbalance of the vortical flow from further increase and maintaining approximately a balanced flow.

It is crucial to make clear how the proposed point of view could improve the classical description of convection. The key point is that, by introducing the framework of balanced flows, it extends previous theories which attribute convection mainly to dynamical instabilities of the balanced basic state. The presented theory considers not only the apparent instabilities but also the interaction of convective activities with the imbalanced basic state. Moreover, the basic state can now be much more complex than in traditional theory. These differences need not to increase the difficulties in the analysis of the apparent instability and the interaction when $\ell_{5, \phi} \delta$ is dropped just as in Sect. 3 .

The interaction between convection and basic flow is the typical situation for dry or adiabatic convection. Although we suppose that these purely dynamical processes work also for moist or diabatic convection, other important processes contributing to the interaction are associated with the existence of water vapor. The dominant thinking about the interaction between large-scale atmospheric circulation and moist convection holds that convection acts as a heat source for large-scale circulation, while the latter supplies water vapor to the convection. Emanuel et al. (1994) shows that this idea has led to fundamental misconceptions and offers an alternative paradigm. They suggest that the understanding of largescale circulations in convecting atmospheres can be regarded as a problem of understanding the distribution in space and time of the subcloud-layer entropy to which the temperature profile is directly tied. Also, they argue that the direct effect of convection on large-scale circulations is to reduce by roughly an order of magnitude the effective static stability felt by such circulations, and to damp all of them. We believe that our results do not conflict with those of Emanuel et al. (1994), because these two theories just describe two different aspects (i.e. convections with and without water vapor considered) of interaction between convection and its basic state. Emanuel et al. (1994) also argue that the respective time scales of convection and larger-scale forcing are too 
disparate to allow convection to directly affect the largerscale flow. This is a central assumption in the statistical quasi-equilibrium hypothesis (SQE), which is not opposite to our conjecture that convection serves to adjust the largerscale environment to a state of balance. What Emanuel et al., mean by convection corresponds just to the free modes of convection or the homogeneous solution that is not related to the vortical flows in the linear stage of growth. On the contrary, the forced part of convection or the inhomogeneous solution does serve to adjust the larger-scale environment as was pointed in last section. Such a discrepancy is just due to the difference of definitions of convection and is not significant for larger-scale vortical flow. But for smaller-scale vortical flows with strong imbalance, this discrepancy may become important.

Another question may also arise from this difference of definitions of convection, that is, the larger-scale environment always has regions of convergence/divergence of the same scale, while a vortical flow associated with the forcing $\mathfrak{R}(\varsigma, \phi)$ is always nondivergent. This can simply be explained because in the generalized definition these largerscale convergence/divergence is included in the convection rather than in its environment. It is also easy to see that the friction and diabatic heating can be incorporated into the present framework without technical difficulty. As a result, the effects of the physical boundary layer and latent heating on convections can be discussed within this framework as well, which will be the topic of our future investigations on this issue. We believe these theoretical results on balanced circulations with convective activity can provide a new perspective for diagnostic studies to understand the formation and the structure of meso-scale convection systems.

\section{Appendix A}

Let $L$ denote the linear operator in the left-hand side of (15), i.e.

$L=-\frac{d^{2}}{d p^{2}}$

Then the eigen-system (15) can be written as

$L P_{n}=\lambda_{n} \sigma P_{n} ; \quad n=0,1,2, \ldots$

It may satisfy the following boundary conditions of the first, second and third kinds at $p=0$ and $p_{s}$ :

$\alpha_{1} P_{n}(0)+\beta_{1} P_{n}^{\prime}(0)=0$

$\alpha_{2} P_{n}\left(p_{s}\right)+\beta_{2} P_{n}^{\prime}\left(p_{s}\right)=0$.

Here, $\left|\alpha_{1}\right|^{2}+\left|\beta_{1}\right|^{2} \neq 0 ;\left|\alpha_{2}\right|^{2}+\left|\beta_{2}\right|^{2} \neq 0$. We define the inner product by

$<u, v>=\int_{0}^{p_{s}} u^{*} v d p$
It is easy to prove that

$<P_{n}, L P_{n}>-<L P_{n}, P_{n}>=\left(\lambda_{n}-\lambda_{n}^{*}\right) \int_{0}^{p_{0}} \sigma\left|P_{n}\right|^{2} d p$.

And by multiplying Eq. (A2) by $P_{n}^{*}$, we have also

$\lambda_{n} \int_{0}^{p_{0}} \sigma\left|P_{n}\right|^{2} d p=-\left.P_{n} \frac{d P_{n}^{*}}{d p}\right|_{0} ^{p_{0}}+\int_{0}^{p_{0}}\left|\frac{d P_{n}}{d p}\right|^{2} d p$.

Since $L$ is a self-adjoint operator and the first term of the right hand side of Eq. (A6) is non-negative under any of the boundary conditions of Eq. (A3), for an arbitrary nontrivial solution of Eq. (A2), we have

$\left(\lambda_{n}-\lambda_{n}^{*}\right) \int_{0}^{p_{0}} \sigma\left|P_{n}\right|^{2} d p=0$

$\lambda_{n} \int_{0}^{p_{0}} \sigma\left|P_{n}\right|^{2} d p>0$.

For a certain eigenfunction $P_{n}$, we can define an equivalent parameter of static stability by the weighted average of $\sigma$ over the entire layer of the atmosphere, i.e.

$\sigma_{n}=\int_{0}^{p_{0}} \sigma\left|P_{n}\right|^{2} d p$

where the weight is chosen as the power of the normalized $P_{n}$. It can be inferred from Eq. (A7a and b) that, if $\sigma_{n}>0$ $\left(\sigma_{n}<0\right), \lambda_{n}$ is real and $\lambda_{n}>0\left(\lambda_{n}<0\right)$. A special case is when $\sigma>0(\sigma<0)$ at all levels. In other words, if the atmosphere is statically stable, then $\lambda_{n}>0$ holds for all eigenfunctions. Otherwise, if the atmosphere is statically unstable at some layer, it is assumed that we can find some $\lambda_{n}<0$, a particular case of which is all the $\lambda_{n}<0$ when $\sigma<0$, or the atmosphere is statically unstable at the whole layer.

It is necessary to specify the vertical boundary condition suitable for the present issue from Eq. (A3). By letting $\alpha_{1}=0$ and $\beta_{2} / \alpha_{2}>0$ in Eq. (A3), the eigen-system satisfies the second and the third kind of boundary conditions at $p=0$ and $p_{s}$, respectively. The physical meanings of these boundary conditions are also clear: there is no exchange of convergence at the top of the atmosphere, and exchange of convergence at surface is proportional to the convergence in situ.

Acknowledgements. The authors are grateful to R. Donner and two anonymous referees for their insightful comments and suggestions. This study is supported by the National Natural Science Foundation of China under Grant No. 41175065 and 41075039 and, the Chinese Special Scientific Research Project for Public Interest under Grant No. GYHY200806009, GYHY201206038, and the Qinglan Project of Jiangsu Province of China under Grant No. 2009.

Edited by: R. Donner

Reviewed by: two anonymous referees 


\section{References}

Arakawa, A. and Schubert, W. H.: Interaction of a cumulus cloud ensemble with the large-scale environment, Part I., J. Atmos. Sci., 31, 674-701, 1974.

Charney, J. G.: The use of the primitive equation of motion in numerical prediction, Tellus, 7 22-26, 1955.

Drazin, P. G.: Hydrodynamical stability, Cambridge University Press, Cambridge, 1981.

Emanuel, K. A., Neelin, J. D., and Bretherton, C. S.: On large-scale circulations in convecting atmospheres, Quart. J. Roy. Met. Soc., 120, 1111-1143, 1994.

Ford, R., McIntyre, M. E., and Norton, W. A.: Balance and the slow quasimanifold: Some explicit results, J. Atmos. Sci., 57, 12361254, 2000.

Guo, D. R.: Methods of Mathematical Physics, People's Education Press, Beijing, 1979 (in Chinese).

Haberman, R.: Applied Partial Differential Equations, 4th Edn., Prentice Hall, 2003.

Holton J. R.: An introduction to dynamic meteorology, Academic Press, 1992.

Hoskins, B. J.: The role of potential vorticity in symmetric stability and instability, Quart. J. Roy. Meteor. Soc, 100, 480-482, 1974.

Jacobs, S. J.: On the existence of a slow manifold in a model system of equations, J. Atmos. Sci., 48, 793-801, 1991.

Leith, C. E.: Nonlinear normal mode initialization and quasigeostrophic theory, J. Atmos. Sci., 37, 958-968, 1980.

Lighthill, M. J.: On sound generated aerodynamically, I. General theory, Proc. Roy. Soc. London, Ser. A, Math. Phys. Sci., 211, 564-587, 1952.

Lorenz, E. N.: Attractor sets and quasi-geostrophic equilibrium, J. Atmos. Sci., 37, 1685-1699, 1980.

Lorenz, E. N.: On the existence of a slow manifold, J. Atmos. Sci., 43, 1547-1557, 1986.

Lorenz, E. N. and Krishnamurthy, V.: On the nonexistence of a slow manifold, J. Atmos. Sci., 44, 2940-2950, 1987.

Lorenz, E. N.: The Slow Manifold - What Is It?, J. Atmos. Sci., 49, 2449-2451, 1992.
Lynch, P.: The slow equations, Q. J. Roy. Meteor. Soc., 115, 201219, 1989.

McIntyre, M. E.: Balance, potential-vorticity inversion, Lighthill radiation, and the slow quasimanifold, Proc. IUTAM/IUGG/Royal Irish Academy Symposium on Advanced in Mathematical Modelling of Atmosphere and Ocean held at the Univ. of Limerick, Ireland, 2-7 July 2000, edited by: Hodnett, P. F., 45-68, 2000.

Moura, A. D.: The Eigensolutions of the Linearized Balance Equations over a Sphere, J. Atmos. Sci., 33, 877-907, 1976.

Pedlosky, J.: Geophysical Fluid Dynamics, Springer Verlag, New York, 1979.

de Roode, S. R., Duynkerke, P. G., and Jonker, H. J. J.: Large eddy simulation: How large is large enough?, J. Atmos. Sci., 61, 403421, 2004.

Vallis, G. K.: Potential vorticity and balanced equation of motion for rotating and stratified flows, Q. J. Roy. Met. Soc., 122, 291322, 1996.

Vanneste, J. and Yavneh, I.: Exponentially Small Inertia-Gravity Waves and the Breakdown of Quasigeostrophic Balance, J. Atmos. Sci., 61, 211-223, 2004.

Vautard, R. and Legras, B.: Invariant manifolds, quasi-geostrophy and initialization, J. Atmos. Sci., 43, 565-584, 1986.

Warn, T.: Nonlinear balance and quasi-geostrophic sets, Atmos. Ocean., 35, 135-145, 1997.

Warn, T. and Menard, R.: Nonlinear balance and gravity-inertia wave saturation in a simple atmospheric model, Tellus, 38A, 285-294, 1986.

Warn, T., Bokhove, O., Shepherd, T. G., and Vallis, G. K.: Rossby number expansions, slaving principles, and balance dynamics, $Q$. J. Roy. Met. Soc., 121, 723-739, 1995.

Williams, P. D., Haine, T. W. N., and Read, P. L.: Inertia-Gravity Waves Emitted from Balanced Flow: Observations, Properties, and Consequences, J. Atmos. Sci., 65, 3543-3556, 2008.

$\mathrm{Xu}, \mathrm{Q}$. and Clark, J. H. E.: The Nature of Symmetric Instability and Its Similarity to Convective and Inertial Instability, J. Atmos Sci., 42, 2880-2883, 1985.

Zhao, N.: Criteria for the stability of steady flows induced by diabatic heating, Dyn. Atmos. Oceans., 36, 297-307, 2003. 